\title{
TITLE:
}

\section{Large-scale lognormality in turbulence modeled by the Ornstein-Uhlenbeck process}

$\operatorname{AUTHOR}(S)$ :

Matsumoto, Takeshi; Takaoka, Masanori

\section{CITATION:}

Matsumoto, Takeshi ...[et al]. Large-scale lognormality in turbulence modeled by the Ornstein-Uhlenbeck process. Physical Review E 2013, 87(1): 013008.

ISSUE DATE:

2013-01

URL:

http://hdl.handle.net/2433/171239

RIGHT:

C)2013 American Physical Society 
PHYSICAL REVIEW E 87, 013008 (2013)

\title{
Large-scale lognormality in turbulence modeled by the Ornstein-Uhlenbeck process
}

\author{
Takeshi Matsumoto* \\ Division of Physics and Astronomy, Graduate School of Science, Kyoto University, Kitashirakawa Oiwakecho Sakyoku, \\ Kyoto 606-8502, Japan \\ Masanori Takaoka \\ Department of Mechanical Engineering, Doshisha University, Kyotanabe, Kyoto 610-0321, Japan
}

(Received 14 August 2012; published 8 January 2013)

\begin{abstract}
Lognormality was found experimentally for coarse-grained squared turbulence velocity and velocity increment when the coarsening scale is comparable to the correlation scale of the velocity [Mouri et al., Phys. Fluids 21, 065107 (2009)]. We investigate this large-scale lognormality by using a simple stochastic process with correlation, the Ornstein-Uhlenbeck (OU) process. It is shown that the OU process has a similar large-scale lognormality, which is studied numerically and analytically.
\end{abstract}

DOI: 10.1103/PhysRevE.87.013008

PACS number(s): 47.27.Ak, 47.27.eb

\section{INTRODUCTION}

The lognormal distribution appears in a wide range of natural and social phenomena (see, e.g., [1]). In fluid turbulence, it is well known that the distribution is an important consequence of Kolmogorov's 1962 theory for modeling fluctuations of the energy cascade rate across scales [2]. In this theory, we have a very clear picture why the lognormal distribution was the first candidate for the cascade fluctuations in his refined phenomenology. Namely, the energy cascade at a high Reynolds number can be modeled as a multiplicative process consisting of a large number of independently and identically distributed random variables. This large number is important for the central limit theorem to be applicable to the logarithm of the multiplicand.

There is a different example of lognormally distributed variables in turbulence. Laboratory experiments of turbulent boundary layers in the 1980s suggest that spanwise separations between the low-speed streaks follow a lognormal distribution $[3,4]$. In this case, the underlying mechanism of the lognormally distributed streaks is not as clear as in the Kolmogorov 1962 phenomenology because a multiplicative structure for the streaks is not found immediately.

In this paper we consider yet another lognormal example in turbulence, which was recently found experimentally $[5,6]$. In Ref. [6], it is observed that the coarse-grained squared velocity and squared velocity increments over distance $r$,

$$
\begin{gathered}
u_{R}^{2}\left(x_{c}\right)=\frac{1}{R} \int_{x_{c}-R / 2}^{x_{c}+R / 2} u^{2}(x) d x, \\
\delta u_{r, R}^{2}\left(x_{c}\right)=\frac{1}{R-r} \int_{x_{c}-R / 2}^{x_{c}+R / 2}[u(x+r)-u(x)]^{2} d x,
\end{gathered}
$$

are lognormally distributed when the averaging scale $R$ is set to $R \sim L_{u}$. Here $L_{u}$ is the correlation length, defined as the integral scale of the velocity correlation function $\langle u(x+$ $r) u(x)\rangle$, and the velocity $u$ can be either a longitudinal or a transverse velocity component. Since the lognormal property holds when $R$ is comparable to the large-scale $L_{u}$, this property

\footnotetext{
*takeshi@kyoryu.scphys.kyoto-u.ac.jp
}

is called "large-scale lognormality." For the asymptotic regime $R \gg L_{u}$, as a usual result of the central limit theorem, the distribution of the coarse-grained quantities becomes closer and closer to a normal (Gaussian) distribution. Note that largescale lognormality, which we consider here, concerns the range of the coarse-graining scale $R \sim L_{u}$, which is different from the final Gaussian state in $R \gg L_{u}$.

Large-scale lognormality was observed in the experiments on grid turbulence, turbulent boundary layers, and turbulent jets, suggesting its universality [6]. But again, as in the case of the streaks in the boundary layer turbulence, why the coarsegrained turbulence data are lognormally distributed is not clear. Our final goal is to understand the mechanism that makes them so.

As the first step toward this goal, we address the following question: Is large-scale lognormality a general property of correlated random variables, not restricted to large-scale fluctuations of turbulence? The previous results [6] lead us to recognize that this balance between $R$ and $L$ is most important for lognormality. In order to answer the question, we use the Ornstein-Uhlenbeck (OU) process as the simplest way to generate correlated random variables with correlation length $L$ and check whether or not coarse-grained quantities like Eqs. (1) and (2) follow lognormal distributions in the range $R / L \sim 1$. Our numerical results suggest that the answer to this question is yes. Then we study, by analytical calculations of moments, further details on how the OU data become close to the lognormal variables.

We believe that this simple approach using the stochastic process has some value since reproducing the large-scale lognormality by direct numerical simulations of the NavierStokes equations can be computationally quite expensive. It requires a very large domain size compared with the correlation length.

In statistics, taking the logarithm of a positive random variable is known as a common way of symmetrizing transformation, which makes the skewness of the transformed variable closer to 0 [7]. In this language, the large-scale lognormality suggests that, even with the correlation, the log transformation can already produce near-Gaussian behavior successfully when the averaging scale $R$ is of the order of the correlation scale $L$. The present study can be interpreted 
as a model study on how the log transformation begins to work for the correlated random variable upon changing the coarse-graining scale.

This paper is organized as follows. In Sec. II, we present numerical data on the OU process reproducing the largescale lognormality. We then, in Sec. III, provide analytical calculations of moments of the OU process to study how they are close to those of the lognormal distribution. We provide a summary and discussion in Sec. IV.

\section{NUMERICAL EXPERIMENT ON THE ORNSTEIN-UHLENBECK PROCESS}

We begin here by listing the basic properties of the OU process (see, e.g., [8] and also [9] in the context of turbulence modeling). The process is described by the Langevin equation

$$
\frac{d X(t)}{d t}=-\frac{1}{T_{L}} X(t)+\sqrt{2 \kappa} \xi(t),
$$

where the Langevin noise $\xi(t)$ is Gaussian white noise having the ensemble-averaged mean and variance

$$
\langle\xi(t)\rangle=0, \quad\left\langle\xi(t) \xi\left(t^{\prime}\right)\right\rangle=\delta\left(t-t^{\prime}\right) .
$$

The linear addition of the Gaussian uncorrelated noise $\xi(t)$ causes the solution $X(t)$ to Eq. (3) to be a Gaussian random variable. However, the first term on the right-hand side brings temporal correlation. Namely, by writing the initial position as $x_{0}, X(t)$ is characterized as a correlated-in-time Gaussian random variable with the mean and variance

$$
\begin{gathered}
\langle X(t)\rangle=x_{0} e^{-t / T_{L}}, \\
\left\langle[X(t)-\langle X(t)\rangle]^{2}\right\rangle=\kappa T_{L}\left(1-e^{-2 t / T_{L}}\right) .
\end{gathered}
$$

The correlation function can be calculated analytically as

$$
\begin{aligned}
& \langle[X(t)-\langle X(t)\rangle][X(t+s)-\langle X(t+s)\rangle]\rangle \\
& \quad=\left\langle[X(t)-\langle X(t)\rangle]^{2}\right\rangle e^{-|s| / T_{L}} .
\end{aligned}
$$

Therefore the integral scale of the OU process is given as

$$
\int_{0}^{\infty} \frac{[\langle X(t)-\langle X(t)\rangle][X(t+s)-\langle X(t+s)\rangle]\rangle}{\left\langle[X(t)-\langle X(t)\rangle]^{2}\right\rangle} d s=T_{L} .
$$

If the large-scale lognormality observed in turbulence [6] is a property of correlated random variables, it is then likely that the following coarse-grained data on the OU process,

$$
\begin{gathered}
X_{R}^{2}(t)=\frac{1}{R} \int_{t-R / 2}^{t+R / 2} X(t)^{2} d t, \\
\delta X_{r, R}^{2}(t)=\frac{1}{R-r} \int_{t-R / 2}^{t+R / 2}[X(t+r)-X(t)]^{2} d t,
\end{gathered}
$$

are lognormally distributed when the averaging scale $R$ is in the similar range, such as $1 \leqslant R / T_{L} \leqslant 100$. Equations (9) and (10) correspond to the turbulence quantities, Eqs. (1) and (2), respectively.

We now check whether or not the random variables, (9) and (10), follow lognormal distributions by doing numerical simulation of the OU process, (3). We use the numerical method called the exact updating formula of the OU process proposed in Ref. [10] with the parameters $x_{0}=0.0, T_{L}=1.0$,
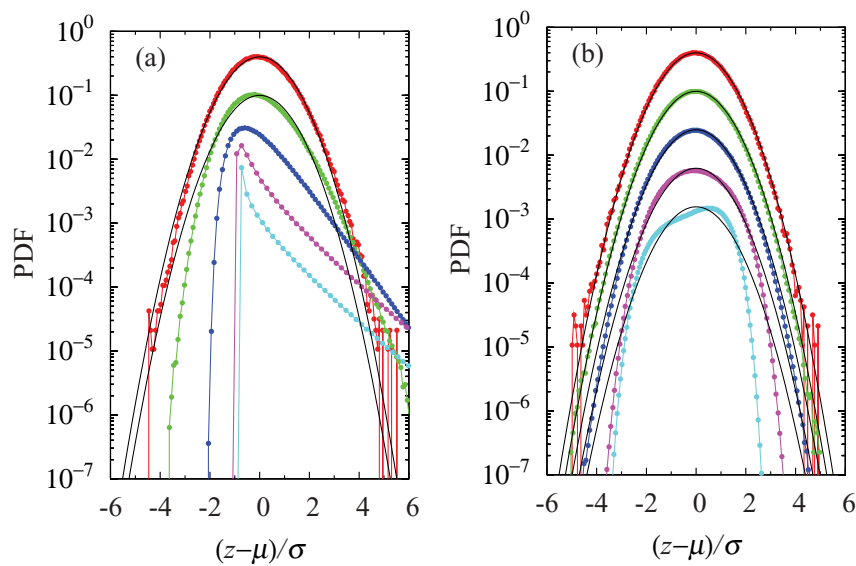

FIG. 1. (Color online) Probability density functions (PDFs) of (a) $X_{R}^{2}$ [Eq. (9)] and (b) its logarithm $\ln X_{R}^{2}$. Random variables are normalized to have zero mean and unit standard deviation. The averaging scales here are $R=1000 T_{L}, 100 T_{L}, 10 T_{L}, T_{L}$, and $T_{L} / 10$, which correspond to the curves from top to bottom. The PDFs are shifted vertically by being multiplied by the factor 0.25 for clarity. The solid curve denotes the Gaussian distribution.

$\kappa=0.50$, and $\Delta t=1.0 \times 10^{-3}$. The integrals in Eqs. (9) and (10) are numerically calculated as

$$
\begin{gathered}
X_{R}^{2}\left(t_{n}\right)=\frac{1}{N_{R}} \sum_{j=-N_{R} / 2}^{N_{R} / 2} X\left(t_{n+j}\right)^{2}, \\
\delta X_{r, R}^{2}\left(t_{n}\right)=\frac{1}{N_{R}} \sum_{j=-N_{R} / 2}^{N_{R} / 2}\left[X\left(t_{n+j}+t_{N_{r}}\right)-X\left(t_{n+j}\right)\right]^{2},
\end{gathered}
$$

where $N_{R}=R / \Delta t, N_{r}=r / \Delta t$, and $t_{k}=k \Delta t$. Figures 1 and 2 show probability density functions (PDFs) of $X_{R}^{2}$ and $\delta X_{r, R}^{2}$ with or without taking the logarithm for various averaging scales $R$. The number of samples for the $R=1000 T_{L}$ case is $1.34 \times 10^{6}$ (for smaller $R$ cases, the number is much larger). For the largest values of $R=1000 T_{L}$ shown here, the PDFs are close to Gaussian distributions as a consequence of the
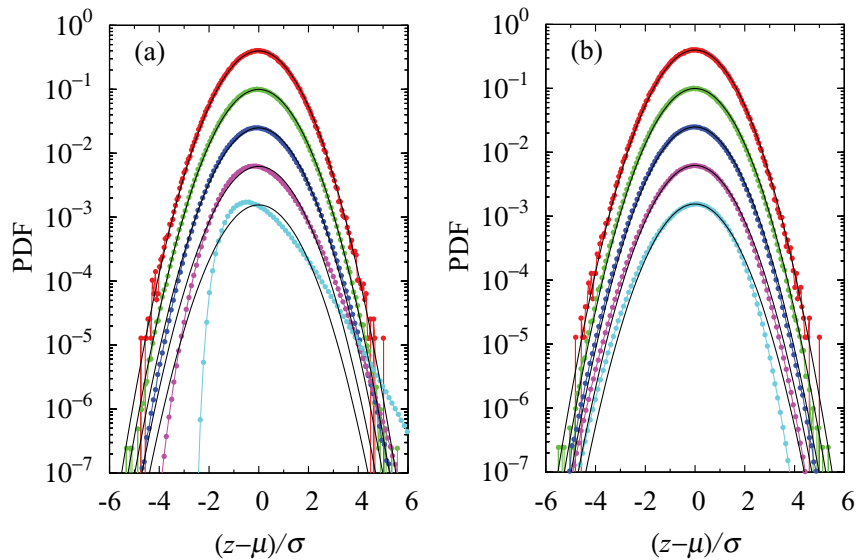

FIG. 2. (Color online) Same as Fig. 1 but for the squared increment (a) $\delta X_{r, R}^{2}$ [Eq. (10)] and (b) its logarithm $\ln X_{r, R}^{2}$. Here $r=T_{L} / 100$. The averaging scales are $R=1000 T_{L}, 100 T_{L}, 10 T_{L}$, $T_{L}$, and $T_{L} / 10$, which correspond to the curves from top to bottom. 

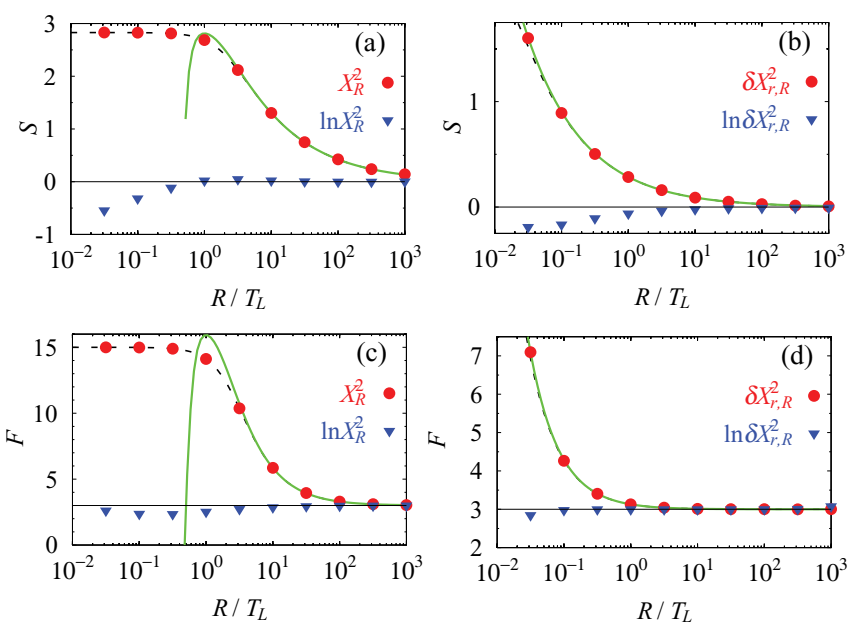

FIG. 3. (Color online) Skewness (a) of $X_{R}^{2}$ and (b) of $\delta X_{r, R}^{2}$ ( $r=T_{L} / 100$ ); flatness (c) of $X_{R}^{2}$ and (d) of $\delta X_{r, R}^{2}$ with or without the logarithm. They are plotted versus the averaging scale $R$. Horizontal lines correspond to the skewness and the flatness of the Gaussian distributions. Thick curves are asymptotic expressions of the moments of $X_{R}^{2}$ and $\delta X_{r, R}^{2}$, Eqs. (25), (26) and Eqs. (28), (29). Dashed curves correspond to the exact moment expressions (not involving any asymptotic argument).

central limit theorem. As we decrease the averaging scale $R$ to the correlation scale $T_{L}$, the distributions of $X_{R}^{2}$ and $\delta X_{r, R}^{2}$ deviate from the Gaussian distribution. In contrast, the log variables $\ln X_{R}^{2}$ and $\ln \delta X_{r, R}^{2}$ remain nearly Gaussian as shown in Figs. 1(b) and 2(b). Hence $X_{R}^{2}$ and $\delta X_{r, R}^{2}$ are lognormally distributed in this range of $R$. This behavior is similar to the turbulence data analyzed in Ref. [6]. In addition, for the squared increments $\delta X_{r, R}^{2}$ with various $r<T_{L}$, qualitatively the same results are obtained.

The approach to the lognormal distribution can be observed more quantitatively by looking at how the skewnesses and flatnesses of the $\log$ variables $\ln X_{R}^{2}$ and $\ln \delta X_{r, R}^{2}$ change as functions of $R$ [the skewness $S(z)$ and flatness $F(z)$ of a random variable $z$ are defined as $S(z)=\left\langle(z-\langle z\rangle)^{3}\right\rangle /[V(z)]^{3 / 2}, F(z)=$ $\left\langle(z-\langle z\rangle)^{4}\right\rangle /[V(z)]^{2}$, where $V(z)$ is the variance $\left.\left\langle(z-\langle z\rangle)^{2}\right\rangle\right]$. In Fig. 3, it is shown that the moments of the variables with logarithm $\ln X_{R}^{2}$ and $\ln \delta X_{r, R}^{2}$ already approach, around $R=$ $T_{L}$, the values of the Gaussian distributions $(S, F)=(0,3)$, whereas the moments of the variables without logarithms are still different from them. This is consistent with the behavior shown in Figs. 1 and 2.

For the increments $\delta X_{r, R}^{2}$, the skewness and the flatness can depend on the difference $r$. Indeed a clear $r$ dependence is seen in Fig. 4. However, the fast convergence to the Gaussian of the $\ln \delta X_{r, R}^{2}$ around $R \sim L$ is not affected by this dependence. In fact, these graphs of the different $r$ values can be collapsed to one curve by normalizing $R$ with a different correlation scale from $T_{L}$, as we show at the end of the next section.

In summary, we observe that the large-scale lognormality holds also for the OU process as in the turbulence case [6]. Further details on the behavior of the skewness and flatness of the OU process are studied analytically in the next section.
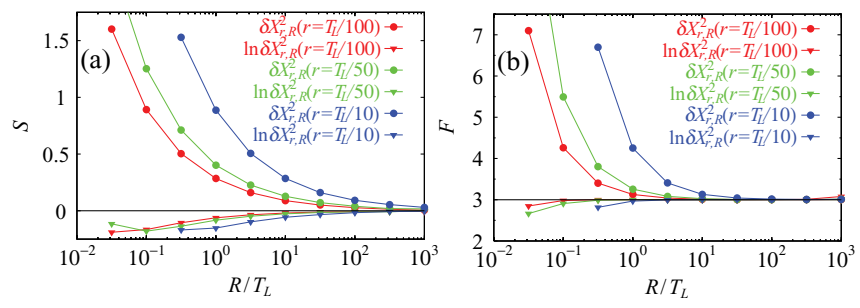

FIG. 4. (Color online) (a) Skewness and (b) flatness of the increments $\delta X_{r, R}^{2}$ and $\ln \delta X_{r, R}^{2}$ for three values of $r: r=T_{L} / 10, T_{L} / 50$, and $T_{L} / 100$.

\section{ANALYTICAL EXPRESSIONS OF THE MOMENTS OF THE ORNSTEIN-UHLENBECK PROCESS}

The OU process is amenable to analytical calculation in many ways. However, it is difficult to determine analytically the PDFs of the quantities of our interest, Eqs. (9) and (10), or all their moments, since they involve integrations. Instead we calculate the low-order moments, namely, the skewness and the flatness of $X(t)_{R}^{2}$ and $\delta X(t)_{r, R}^{2}$. For them, full analytic results are obtained but we present only the asymptotic expressions of them for $R / L \gg 1$ since they are sufficient for our purpose.

Then we compare them with the expressions of the skewness and flatness of the lognormal distribution with mean $E$ and variance $V$ :

$$
\begin{gathered}
S_{\mathrm{LN}}=\sqrt{\rho}(3+\rho), \\
F_{\mathrm{LN}}=3+\rho\left(16+15 \rho+6 \rho^{2}+\rho^{3}\right),
\end{gathered}
$$

where $\rho=V / E^{2}$. As we have seen, the PDFs of the variables $X(t)_{R}^{2}$ and $\delta X(t)_{r, R}^{2}$ become close to the lognormal distributions for $R / T_{L} \sim 1$. We study this behavior by comparing the skewness and flatness of $X(t)_{R}^{2}$ and $\delta X(t)_{r, R}^{2}$ with $S_{\mathrm{LN}}$ and $F_{\mathrm{LN}}$. However, as we shall see below, it turns out that small corrections exist, which may be hard to detect in numerical calculations in the previous section.

In our analytical calculation of the moments, we first put the formal solution of the Langevin equation, (3),

$$
X(t)=e^{-t / T_{L}} x_{0}+\int_{0}^{t} e^{-(t-s) / T_{L}} \sqrt{2 \kappa} \xi(s) d s,
$$

into the coarse-grained variables, Eqs.(9) and (10). For the correlation or the variance, we take suitable powers of them and take the ensemble average with respect to the Langevin noise by using Eq. (4), so that we can reduce their calculations into multiple integrals of certain exponential functions. For the skewness and the flatness, we use more compact integral representations proposed in Ref. [11], which are explained in the Appendix. To calculate the resultant integrals we use a symbolic computation software, Maple. The integrated result still contains dependence on time $t$. For example, the mean of $X(t)_{R}^{2}$ reads

$$
\left\langle X(t)_{R}^{2}\right\rangle=\kappa T_{L}\left[1+\frac{T_{L}}{2 R}\left(e^{-R / T_{L}}-e^{R / T_{L}}\right) e^{-2 t / T_{L}}\right] .
$$

Remember that this time $t$ is much larger than the correlation time $T_{L}$ to be consistent with the simulations in Sec. II. We hence regard $e^{-t / T_{L}}=0$. From now on, we drop the dependency on $t$ of all the moments of $X(t)_{R}^{2}$ and $\delta X(t)_{r, R}^{2}$ 
calculated here. This does not affect our study since the OU process becomes steady for $t \gg T_{L}$.

We now list the expressions of the moments. Here we write $\Lambda=R / T_{L}$ for brevity. For $X_{R}^{2}$, the mean, variance, skewness, and flatness are, respectively,

$$
\begin{gathered}
E\left(X_{R}^{2}\right)=\kappa T_{L}, \\
V\left(X_{R}^{2}\right)=\left(\kappa T_{L}\right)^{2}\left[2 \Lambda^{-1}+\Lambda^{-2}\left(e^{-2 \Lambda}-1\right)\right], \\
S\left(X_{R}^{2}\right)=12 W_{0}^{-3 / 2}\left[e^{-2 \Lambda}-1+\Lambda\left(e^{-2 \Lambda}+1\right)\right], \\
F\left(X_{R}^{2}\right)=3+6 W_{0}^{-2}\left[e^{-4 \Lambda}+28 e^{-2 \Lambda}-29\right. \\
\left.+16 \Lambda^{2} e^{-2 \Lambda}+40 \Lambda e^{-2 \Lambda}+20 \Lambda\right],
\end{gathered}
$$

where $W_{0}=2 \Lambda^{-1}+\Lambda^{-2}\left(e^{-2 \Lambda}-1\right)$. For the coarse-grained increment $\delta X_{r, R}^{2}$, by writing $r / T_{L}=\lambda$, we give the results in the leading order of $\Lambda^{-1}=\left(R / T_{L}\right)^{-1}$ to avoid lengthy expressions:

$$
\begin{gathered}
E\left(\delta X_{r, R}^{2}\right)=2 \kappa T_{L} e^{-\lambda}\left(e^{\lambda}-1\right), \\
V\left(\delta X_{r, R}^{2}\right)=\left(2 \kappa T_{L}\right)^{2} \Lambda^{-1} e^{-2 \lambda}\left[3 e^{2 \lambda}-4(\lambda+1) e^{\lambda}+2 \lambda+1\right],
\end{gathered}
$$

$$
\begin{aligned}
S\left(\delta X_{r, R}^{2}\right)= & 3 \Lambda^{-2} e^{-3 \lambda} W_{1}^{-3 / 2}\left[10 e^{3 \lambda}-5\left(\lambda^{2}+3 \lambda+3\right) e^{2 \lambda}\right. \\
& \left.+2\left(4 \lambda^{2}+6 \lambda+3\right) e^{\lambda}-\left(3 \lambda^{2}+3 \lambda+1\right)\right], \quad(23) \\
F\left(\delta X_{r, R}^{2}\right)= & 3+\Lambda^{-3} e^{-4 \lambda} W_{1}^{-2}\left[525 e^{4 \lambda}-\left(56 \lambda^{3}+336 \lambda^{2}\right.\right. \\
& +840 \lambda+840) e^{3 \lambda}+\left(224 \lambda^{3}+672 \lambda^{2}+840 \lambda\right. \\
& +420) e^{2 \lambda}-\left(216 \lambda^{3}+432 \lambda^{2}+360 \lambda\right. \\
& \left.+120) e^{\lambda}+\left(64 \lambda^{3}+96 \lambda^{2}+60 \lambda+15\right)\right],
\end{aligned}
$$

where $W_{1}=\Lambda^{-1} e^{-2 \lambda}\left[3 e^{2 \lambda}-4(\lambda+1) e^{\lambda}+2 \lambda+1\right]$. Note that we obtain analytic expressions of $S$ and $F$, which are shown as the dashed curves in Fig. 3.

With these asymptotic expressions, we next rewrite the skewness $S$ and flatness $F$ as functions of the variance over the squared mean $\rho=V / E^{2}$ to compare them with the lognormal ones $S_{\mathrm{LN}}$ and $F_{\mathrm{LN}}$.

For $X_{R}^{2}$, in the limits of $\Lambda=R / T_{L} \rightarrow \infty$, we have

$$
\begin{gathered}
S\left(X_{R}^{2}\right) \simeq 0+\sqrt{\rho}\left(3-\frac{3}{16} \rho^{2}\right), \\
F\left(X_{R}^{2}\right) \simeq 3+\rho\left(15+\frac{3}{8} \rho-\frac{39}{16} \rho^{2}\right),
\end{gathered}
$$

where

$$
\rho=\frac{V\left(X_{R}^{2}\right)}{E\left(X_{R}^{2}\right)^{2}} \simeq \frac{2 T_{L}}{R}-\left(\frac{T_{L}}{R}\right)^{2} .
$$

The asymptotic expressions, Eqs. (25) and (26), agree with data shown in Fig. 3 for $R \geqslant \sqrt{10} T_{L}$.

For the velocity increments $\delta X_{r, R}^{2}$, we have $\rho=V / E^{2}=$ $\Lambda^{-1}\left[3 e^{2 \lambda}-4(\lambda+1) e^{\lambda}+2 \lambda+1\right] /\left(e^{\lambda}-1\right)^{2}$. By taking the limit of $\lambda=r / T_{L} \rightarrow 0$, we obtain the expressions

$$
\begin{aligned}
& S\left(\delta X_{r, R}^{2}\right) \simeq 0+\sqrt{\rho} \times \frac{99}{40}, \\
& F\left(\delta X_{r, R}^{2}\right) \simeq 3+\rho \times \frac{1359}{140},
\end{aligned}
$$

where

$$
\rho=\frac{V\left(\delta X_{r, R}^{2}\right)}{E\left(\delta X_{r, R}^{2}\right)^{2}} \simeq \frac{4 \lambda}{3 \Lambda}=\frac{4 r}{3 R} .
$$

Equations (28) and (29) agree with the data shown in Fig. 3 for $R \geqslant 10^{-3 / 2} T_{L}$ (covering all data points), thanks to the small value $r=10^{-2} T_{L}$.

We now compare the analytic expressions of the OU quantities Eqs. (25), (26) and Eqs. (28), (29) with the lognormal ones, Eqs. (13) and (14). We can observe the following. (i) Indeed as $R \rightarrow \infty(\rho \rightarrow 0), S$ and $F$ of both $X_{R}^{2}$ and $\delta X_{r, R}^{2}$ tend to the values of Gaussian distribution, $S=0$ and $F=3$. Before reaching this state, we see an approach to the lognormal distribution. (ii) The subleading terms of $S$ and $F$ have the same powers of $\rho$ as $S_{\mathrm{LN}}$ and $F_{\mathrm{LN}}$, which is in favor of the large-scale lognormality of the OU process. However, the constant in the subleading term is slightly different from those of the lognormal distribution. In this sense, the coarsegrained quantities $X_{R}^{2}$ and $\delta X_{r, R}^{2}$ of the OU process become nearly lognormally distributed when $R / T_{L}$ is large, but not exactly so.

So far we have focused on how the moments vary as a function of $R / T_{L}$, where $R$ is normalized by the correlation scale $T_{L}$ of the OU process. We close this section with a digression by pointing out another normalization scale of the problem. This is indicated by the above analytic results. For $X_{R}^{2}$, let us go back to the definition, Eq. (9). The integral scale of the integrand of Eq. (9) is

$$
\tau\left(X^{2}\right)=\frac{\int_{0}^{\infty}\left\langle\left[X^{2}(t)-\left\langle X^{2}\right\rangle\right]\left[X^{2}(t+s)-\left\langle X^{2}\right\rangle\right]\right\rangle d s}{\left\langle\left[X^{2}(t)-\left\langle X^{2}\right\rangle\right]^{2}\right\rangle}=\frac{T_{L}}{2} .
$$

For $\delta X_{r, R}^{2}$, the integral scale of its integrand defined similarly is calculated as

$$
\tau\left(\delta X_{r}^{2}\right)=T_{L} \frac{3 e^{2 \lambda}-4(\lambda+1) e^{\lambda}+2 \lambda+1}{4\left(e^{\lambda}-1\right)^{2}} \simeq \frac{r}{3}
$$

for small $\lambda=r / T_{L}$. Note that the $\rho$ variables defined in Eqs. (27) and (30) contain the corresponding integral scales of the integrands (31) and (32), namely, $\rho=4 \tau / R$ for both $X_{R}^{2}$ and $\delta X_{r, R}^{2}$. Hence it implies that suitable normalization scales of $R$ for collapsing the moment data plotted in Figs. 3 and 4 are, respectively, the integral scales $\tau\left(X^{2}\right)$ and $\tau\left(\delta X_{r}^{2}\right)$ of the integrands. Indeed this normalization yields a better collapse as shown in Fig. 5 for both $X_{R}^{2}$ and $\delta X_{r, R}^{2}$ with different $r$. This suggests that, when we look into a coarse-grained quantity
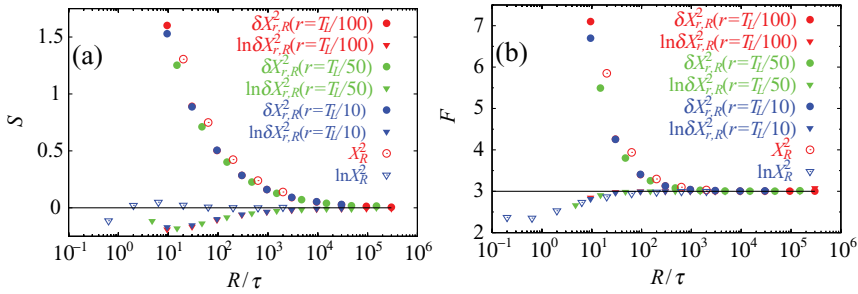

FIG. 5. (Color online) (a) Collapsed skewness and (b) flatness of $X_{R}^{2}$ and $\delta X_{r, R}^{2}$ as functions of $R$ normalized with $\tau\left(X^{2}\right)$ and $\tau\left(\delta X_{r}^{2}\right)$ defined in (31) and (32). 
over scale $R$ of a function $f_{R}(y)=(1 / R) \int_{y}^{y+R} f\left(y^{\prime}\right) d y^{\prime}$ of a correlated fluctuation $y$, the correlation scale (integral scale) of $f(y)$ in certain cases can be a better characteristic scale than the correlation scale of the fluctuation $y$ itself. We believe that this is the case not only for the OU process but also for a general fluctuation with a correlation. However, this normalization blurs the fact that the large-scale lognormality of the OU process occurs at $R / T_{L} \sim 1$. This kind of normalization will be reported elsewhere.

\section{SUMMARY AND DISCUSSION}

This study was motivated by the large-scale lognormality of turbulence that was recently observed experimentally in grid, boundary-layer, and jet turbulences [6]. In this lognormality, the correlation scale plays a pivotal role. Namely, when the averaging scale to the correlation scale is of order unity, the averaged squared velocity and velocity increments become lognormally distributed fluctuations. We anticipated here that this large-scale lognormality is a property of correlated random variables in general.

To test this idea, we took the OU process as the simplest means to generate correlated random variables. Our numerical simulation indicates that the OU process also exhibits large-scale lognormality for about the same range of $R / L$ as observed in turbulence. Then, to further investigate the approach to the lognormal distribution, we calculated analytically the skewness and flatness of the coarse-grained quantities of the OU process. The first subleading term of the asymptotic expressions for large $R / L$ of the moments revealed that indeed the moments behave nearly as those of the lognormal distribution. However, there is small deviation from the lognormal distribution.

Now we speculate on the behavior of the moments that are higher than fourth order. The analytical calculation of them for coarse-grained OU variables becomes so complicated that we did not try even with Maple. For the lognormal variable $x_{\mathrm{LN}}$, the moments can be written with $\rho$, the ratio of the variance to the squared mean, as

$$
\begin{aligned}
H_{\mathrm{LN}}^{(q)} & =\frac{\left\langle\left[x_{\mathrm{LN}}-\left\langle x_{\mathrm{LN}}\right\rangle\right]^{q}\right\rangle}{\left[V\left(x_{\mathrm{LN}}\right)\right]^{q / 2}} \\
& =\rho^{-\frac{q}{2}} \sum_{k=0}^{q}\left(\begin{array}{l}
q \\
k
\end{array}\right)(\rho+1)^{\frac{1}{2} k(k-1)}(-1)^{q-k},
\end{aligned}
$$

where $\left(\begin{array}{l}q \\ k\end{array}\right)$ is the binomial coefficients. By expanding Eq. (33) with $\rho$, the moment can be written as

$$
\begin{aligned}
& H_{\mathrm{LN}}^{(q)} \\
& = \begin{cases}0+s_{1}^{(q)} \rho^{\frac{1}{2}}+s_{2}^{(q)} \rho^{\frac{3}{2}}+\cdots+\rho^{\frac{1}{2} q(q-2)} & (q \text { : odd }), \\
(q-1) ! !+f_{1}^{(q)} \rho+f_{2}^{(q)} \rho^{2}+\cdots+\rho^{\frac{1}{2} q(q-2)} & (q \text { : even }) .\end{cases}
\end{aligned}
$$

Here the terms of the negative powers of $\rho$ are shown to vanish and $s_{1}^{(q)}, f_{1}^{(q)}, \ldots$ are suitable coefficients, which can be written with the binomial coefficients. The $\rho^{0}$ terms in Eq. (34), namely, 0 and $(q-1) ! !=(q-1)(q-3) \cdots$ $5 \cdot 3 \cdot 1$, correspond to the values of the Gaussian distribution. We speculate that, for the coarse-grained quantities of the
OU variable, their higher-order moments have an expression similar to Eq. (34). As we saw in the previous section, the behavior for small $\rho$ (corresponding to $R / T_{L}$ being larger than unity) is relevant for the large-scale lognormality. Their first term should coincide with that of Eq. (34) because of the usual central limit theorem. It is tempting to speculate also that the second term has the same power of $\rho$ but the value of the the coefficient, $s_{1}^{(q)}$ or $f_{1}^{(q)}$, is different. We have no idea by how much they differ.

In relation to the real turbulence data, the OU process is not a good representation as a whole since, for example, it does not deviate from being Gaussian (intermittency effect) and does not show equivalence of the energy cascade or the energy dissipation rate. However, as long as we focus on the large-scale fluctuations of turbulence where the single-point velocity or the velocity increments are close to being Gaussian, the OU process is a useful and analytically tractable model. In this study we regarded that the correlation at the integral scale is the most important aspect to be modeled by the OU process.

Here we have seen that the correlation plays an essential role in the near-lognormal behavior of the coarse-grained positive quantities, Eqs. (9) and (10). Roughly speaking, this near-lognormality around the correlation scale may be regarded as an intermediate state, or "a rule of thumb" before the central limit theorem holds with much larger averaging scale $R$. For the turbulence cases studied in [6], a similar mechanism is likely at work. Other examples of lognormal behavior involving a coarse-graining average include cosmological density fluctuations (see, e.g., [12]), which may have a structure similar to that studied here.

\section{ACKNOWLEDGMENTS}

We gratefully acknowledge discussion with H. Mouri. We were supported by Grant-in-Aid for Scientific Research C No. 22540402 from the Japan Society for the Promotion of Sciences. T.M. was supported by a grant-in-aid for the Global COE Program," The Next Generation of Physics, Spun from Universality and Emergence," from the MEXT of Japan.

\section{APPENDIX}

Here we explain briefly how we calculate the moments of the coarse-grained quantities. In particular, we use Rice's method, given in Sec. 3.9 of Ref. [11], to reduce the number of multiple integrals. For illustration of the method, let us take as an example the second-order moment of $X_{R}^{2}(t)$ :

$$
\left\langle\left[X_{R}^{2}(t)\right]^{2}\right\rangle=\frac{1}{R^{2}} \int_{t-\frac{R}{2}}^{t+\frac{R}{2}} d t_{1} \int_{t-\frac{R}{2}}^{t+\frac{R}{2}} d t_{2}\left\langle X^{2}\left(t_{1}\right) X^{2}\left(t_{2}\right)\right\rangle .
$$

The idea of Rice's method is to write the moment $\left\langle X^{2}\left(t_{1}\right) X^{2}\left(t_{2}\right)\right\rangle$ in terms of the correlation function $\left\langle X\left(t_{1}\right) X\left(t_{2}\right)\right\rangle=\psi\left(t_{2}-t_{1}\right)$ by noting that $X\left(t_{1}\right)$ and $X\left(t_{2}\right)$ are multivariate Gaussian variables whose covariace matrix is known completely. In principle, any moment of $X\left(t_{1}\right), X\left(t_{2}\right), \ldots, X\left(t_{n}\right)$ can be expressed with $\psi\left(t_{i}-t_{j}\right)$. Specifically, with the correlation function for large $t$,

$$
\psi(\tau)=\langle X(t) X(t+\tau)\rangle=\kappa T_{L} e^{-|\tau| / T_{L}},
$$


the variance and other central moments can be written as follows:

$$
\begin{aligned}
& \left\langle\left[X_{R}^{2}(t)-\left\langle X_{R}^{2}\right\rangle\right]^{2}\right\rangle=\frac{2}{R^{2}} \int_{t-\frac{R}{2}}^{t+\frac{R}{2}} d t_{1} \int_{t-\frac{R}{2}}^{t+\frac{R}{2}} d t_{2} \psi^{2}\left(t_{1}-t_{2}\right) \\
& =\frac{4}{R^{2}} \int_{0}^{R}(R-x) \psi^{2}(x) d x \\
& =\frac{\kappa^{2} T_{L}^{2}}{4 R^{2}}\left[2 T_{L} R+T_{L}^{2}\left(e^{-2 R / T_{L}}-1\right)\right], \\
& \left\langle\left[X_{R}^{2}(t)-\left\langle X_{R}^{2}\right\rangle\right]^{3}\right\rangle=\frac{8}{R^{3}} \int_{t-\frac{R}{2}}^{t+\frac{R}{2}} d t_{1} \int_{t-\frac{R}{2}}^{t+\frac{R}{2}} d t_{2} \int_{t-\frac{R}{2}}^{t+\frac{R}{2}} d t_{3} \\
& \times \psi\left(t_{1}-t_{2}\right) \psi\left(t_{2}-t_{3}\right) \psi\left(t_{3}-t_{1}\right) \\
& =\frac{48}{R^{3}} \int_{0}^{R} d x(R-x) \psi(R-x) \\
& \times \int_{0}^{x} d y \psi(x-y) \psi(y), \\
& \left\langle\left[X_{R}^{2}(t)-\left\langle X_{R}^{2}\right\rangle\right]^{4}\right\rangle \\
& =3\left\langle\left(X_{R}^{2}(t)-\left\langle X_{R}^{2}\right\rangle\right)^{2}\right\rangle^{2} \\
& +\frac{48}{R^{4}} \int_{t-\frac{R}{2}}^{t+\frac{R}{2}} d t_{1} \int_{t-\frac{R}{2}}^{t+\frac{R}{2}} d t_{2} \int_{t-\frac{R}{2}}^{t+\frac{R}{2}} d t_{3} \int_{t-\frac{R}{2}}^{t+\frac{R}{2}} d t_{4} \\
& \times \psi\left(t_{2}-t_{1}\right) \psi\left(t_{3}-t_{1}\right) \psi\left(t_{4}-t_{2}\right) \psi\left(t_{4}-t_{3}\right) \\
& =3\left\langle\left(X_{R}^{2}(t)-\left\langle X_{R}^{2}\right\rangle\right)^{2}\right\rangle^{2} \\
& +\frac{768}{R^{4}} \int_{0}^{R} d x(R-x) \int_{0}^{R} d y \psi(y) \psi(x-y) \\
& \times \int_{0}^{y} d z \psi(x-z) \psi(z)
\end{aligned}
$$

[1] E. Limpert, W. A. Stahel, and M. Abbt, BioScience 51, 341 (2001).

[2] A. N. Kolmogorov, J. Fluid Mech. 13, 82 (1962).

[3] H. Nakagawa and I. Nezu, J. Fluid Mech. 104, 1 (1981).

[4] C. R. Smith and S. P. Metzler, J. Fluid Mech. 129, 27 (1983).

[5] H. Mouri, A. Hori, and M. Takaoka, Phys. Fluids 20, 035108 (2008).

[6] H. Mouri, A. Hori, and M. Takaoka, Phys. Fluids 21, 065107 (2009).
Here we change variables in the integrals by using the symmetry of $\psi$ to reduce the double integral to a single integral (for details, see [11]). The integrals, (A4) and (A5), are calculated analytically with the software Maple.

Concerning the increments, we write its correlation function for large $t$ :

$$
\begin{aligned}
\psi_{r}(\tau) & =\left\langle\delta_{r} X(t+\tau) \delta_{r} X(t)\right\rangle \\
& =\kappa T_{L}\left[e^{-|\tau| / T_{L}}\left(2-e^{-r / T_{L}}\right)-e^{-|r-| \tau|| / T_{L}}\right]
\end{aligned}
$$

Using the same argument as in the case of $X_{R}(t)$, we can express the central moments with the correlation $\psi_{r}$ as follows:

$$
\begin{aligned}
\left\langle\left[\delta X_{r, R}^{2}(t)-\left\langle\delta X_{r, R}^{2}(t)\right\rangle\right]^{2}\right\rangle= & \frac{4}{(R-r)^{2}} \int_{0}^{R-r}(R-r-x) \\
& \times \psi_{r}^{2}(x) d x \\
\left\langle\left[\delta X_{r, R}^{2}(t)-\left\langle\delta X_{r, R}^{2}(t)\right\rangle\right]^{3}\right\rangle= & \frac{48}{(R-r)^{3}} \int_{0}^{R-r} d x(R-r-x) \\
& \times \int_{0}^{x} d y \psi_{r}(x-y) \psi_{r}(x) \psi_{r}(y),
\end{aligned}
$$

$$
\begin{aligned}
& \left\langle\left[\delta X_{r, R}^{2}(t)-\left\langle\delta X_{r, R}^{2}(t)\right\rangle\right]^{4}\right\rangle \\
& =3\left\langle\left[\delta X_{r, R}^{2}(t)-\left\langle\delta X_{r, R}^{2}(t)\right\rangle\right]^{2}\right\rangle^{2} \\
& \quad+\frac{768}{(R-r)^{4}} \int_{0}^{R-r} d x(R-r-x) \\
& \quad \times \int_{0}^{R-r} d y \psi_{r}(y) \psi_{r}(x-y) \int_{0}^{y} d z \psi_{r}(x-z) \psi_{r}(z) .
\end{aligned}
$$

The final forms, (A7) and (A8), are calculated with Maple.
[7] A. Das Gupta, Asymptotic Theory of Statistics and Probability (Springer, Berlin, 2008).

[8] C. Gardiner, Stochastic Methods: A Handbook for the Natural and Social Sciences, 4th ed. (Springer, Berlin, 2009).

[9] S. B. Pope, Turbulent Flows (Cambridge University Press, Cambridge, 2000).

[10] D. T. Gillespie, Phys. Rev. E 54, 2084 (1996).

[11] S. O. Rice, Bell System Tech. J. 23, 24, Reprinted in Selected Papers on Noise and Stochastic Processes, edited by N. Wax (Dover, Mineola, NY, 1954).

[12] V. Sahni and P. Coles, Phys. Rep. 262, 1 (1995). 